\title{
Arsenic trioxide induced apoptosis in retinoblastoma cells by abnormal expression of microRNA-376a
}

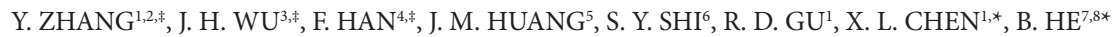 \\ ${ }^{1}$ Department of Ophthalmology, Shengjing Hospital of China Medical University, Shenyang 110004, China; ${ }^{2}$ Department of Ophthalmonogy, \\ Shenyang Military General Hospital, Shenyang 110016, China; ${ }^{3}$ Department of Ophthalmolog, Changhai Hospital, Shanghai 200433, China; \\ ${ }^{4}$ Department of Ophthalmolog, Chengdu Military General Hospital,Chengdu, 610083, China; ${ }^{5}$ Department of Urologic Surgery, 85 Hospital of \\ People's Liberation Army, Shanghai 200052, China; ${ }^{6}$ Department of Ophthalmology, 230 Hospital of People's Liberation Army, Dandong 118000, \\ China; ${ }^{7}$ Department of Cardiothoracic Surgery, Changhai Hospital, Shanghai 200433, China; ${ }^{8}$ Department of Anesthesiology, Xinhua Hospital, \\ Shanghai Jiaotong University School of Medicine, Shanghai 200092, China
}

*Correspondence: cmu_chenxl@yahoo.cn, hebinanes@gmail.com

${ }^{*}$ Contributed equally to this work.

Received August 19, 2012 / Accepted October 2, 2012

\begin{abstract}
Arsenic trioxide (ATO) has been demonstrated to induce apoptosis in retinoblastoma cells, however, mechanisms responsible for this phenomenon are not fully understood. In the present study, we determined whether ATO induced apoptosis by abnormal expression of microRNA. In an apoptosis model of retinoblastoma cells subjected to $4 \mu \mathrm{M}$ ATO for 72 hours, we found 14 miRNAs changed more than 2 -fold by using miRNA microarray analysis. Most of these aberrantly expressed miRNAs were confirmed by quantitative RT-PCR. MiR-376a, a significantly down-regulated miRNA, was selected for further study. The overexpression of miR-376a resulting from miR-376a mimic transfection significantly inhibited ATO-induced apoptosis. By contrast, miR-376a deficiency resulting from miR-376a inhibitor transfection aggravated ATO-induced apoptosis. Using bioinformatic algorithms, caspase-3, a key apoptosis executioner, was predicted as a putative target of miR-376a. The quantitative RT-PCR showed no effects of miR-376a mimic or inhibitor on caspase-3 mRNA level. However, the amount of caspase- 3 proteins was reduced by miR-376a mimic, whereas increased by miR-376a inhibitor. Furthermore, the luciferase reporter assay confirmed caspase- 3 to be a target of miR-376a, and the apoptosis caused by miR-376a inhibitor were abolished by a caspase- 3 inhibitor. These results suggest that ATO -induced apoptosis in retinoblastoma cells is part mediated by decreasing expression of miR-376a, which subsequently increased caspase- 3 expression.
\end{abstract}

Key words: arsenic trioxide, apoptosis, retinoblastoma, microRNA

Retinoblastoma is the third most common form of cancer in infants, and metastatic retinoblastoma is lethal in approximately $90 \%$ of cases. Early detection and aggressive therapy has resulted in a $95 \%$ probability of survival for retinoblastoma patients in the United States [1]. However, the United States represents only 3-4\% of the retinoblastoma cases worldwide. The majority of children diagnosed with retinoblastoma each year live in developing countries where the probability of survival is closer to $50 \%$. This difference in survival rates reflects poor early detection rates and limited resources for the aggressive therapy necessary to treat retinoblastoma and manage the side effects associated with broad-spectrum systemic chemotherapy in young children. In order to have the most significant impact on retinoblastoma treatment, current efforts have been focused on reducing the side effects associated with systemic broad-spectrum chemotherapy.

Arsenic trioxide (ATO) is presently the most active single agent in the treatment of acute promyelocytic leukemia (APL) [2]. ATO acts by targeting multiple pathways in APL leading to apoptosis and myeloid differentiation. It induces complete remission without myelosuppression and causes only few adverse effects. Although its mode of action is still not fully understood, ATO is known to induce cell apoptosis via generation of reactive oxygen species and activation of caspases. Several reports have indicated that ATO acts principally by inducing cell apoptosis not only in APL, but in a variety of non-APL cells including myeloma cells, chronic myeloid leukemia cells and cells of immune origin, including B or 
T lymphocytes, macrophages and, more recently, neuroblastoma $[3,4]$. Retinoblastoma shares phenotypic similarities with neuroblastoma, which has been proved by morphologic, cytogenetic, immunohistochemical, biochemical, and in vitro studies $[5,6]$. Furthermore, it has been reported that high dose ATO had antitumor activity for retinoblastoma by promoting apoptosis [7], but its mechanism is still not very clear.

Given that microRNAs (miRNAs) are important in appropriate cellular function for development, tissue maintenance, and physiology, it is not surprising that the deregulation of miRNAs function is associated with disease. In several human cancer types, miRNAs have been implicated as oncogenes and tumor suppressor genes $[8,9]$. Experiments have identified miRNAs tumor suppressors such as miR-15a, 16-1, 17-5p, 143,145 , and let-7 [9]. In human retinoblastoma, differential let-7b miRNA expression and mRNA expression of let-7b targets has been observed [10], so it is very interesting to investigate whether ATO promote apoptosis of retinoblastoma cells by regulating the expression of miRNAs.

\section{Materials and methods}

Cell lines and culture. Human retinoblastoma cell line HXO-RB44 was maintained in RPMI 1640 media, supplemented with $10 \%$ fetal bovine serum (Gibco BRL, Rockville, MD) and $1 \%$ antibiotic-antimycotic solution (Invitrogen, Carlsbad, $\mathrm{CA})$ at $37^{\circ} \mathrm{C}$ in a moist atmosphere of $95 \%$ air and $5 \% \mathrm{CO}_{2}$. The medium was changed every third day. Cultured tumor cells were observed daily under a phase-contrast microscope.

Analysis of cell apoptosis. In the AV/PI staining method, PI distinguishes viable from dead cells based on its exclusion by viable cells. $\mathrm{AV}$ is a common marker for apoptosis due to their high affinity for phosphatidylserine, which is translocated from the inner to the outer leaflet of the plasma membrane in early apoptosis $[11,12]$. The cells were dual-stained with AV and PI, and then analyzed by flow cytometry.
MiRNA-microarray. Total RNA of cells was isolated by using TRIzol reagent, and reverse transcribed according to the manufacturer's instructions (Fermentas, in CA). MiRNA expression profiling was determined by miRNA-microarray analysis (LC Sciences Inc).

Real-time RT-PCR. Real-time RT-PCR was performed to verify the levels of miRNAs. $1 \mu \mathrm{g}$ of total RNA extracted with Trizol (Invitrogen, Shanghai, China) was reverse-transcribed using M-MLV reverse transcriptase with oligo-dT for mRNAs and a special stem-loop primer for a miRNA (Table 1). Realtime quantitative PCR was performed on a Rotor-Gene 3,000 real-time DNA detection system (Corbett Research, Sydney, Australia) using SYBR Green (Qiagen, Shanghai, China) and the primers listed in Table 2. U6 is spliceosome RNA, the abundance of which is similar to small molecular RNA, and used as miRNA internal reference. GAPDH is a house-keeping gene, and used as a common gene as internal reference of caspase-3.

MiRNA transfection experiments. For transfection experiments, retinoblastoma cells were seeded at a density of $2^{\star}$ $10^{4} \mathrm{cells} / \mathrm{cm}^{2}$ in serum-free DMEM, with the addition of transfection agent and RNAs. The mimic of miR-376a was a duplex RNA, in which the sense sequence was the same as miR-376a. The inhibitor of miR-376a was a single RNA sequence exactly complementary to miR-376a. Mock transfection (transfection without RNA) and non-targeting negative control sequences were used as controls.

Construction of luciferase reporter plasmid and analysis of luciferase activity. To examine whether miR-376a regulates the expression of caspase- 3 , a predicted target of miR-376a, the dual luciferase psiCheck2 reporter plasmid (Promega, Madison, WI, USA) was used to generate the reporter plasmid harboring caspase-3 3'-UTR. Briefly, a 307-bp fragment of caspase-3 3'-UTR containing the putative miR-376a binding site was amplified from genomic DNA by PCR, using Xho 1 and Not 1 primers (left Xho 1 primer: ctcgagCGGAG-

Table 1. Primers for reverse-transcription of miRNAs

\begin{tabular}{ll}
\hline MicroRNA & Primers for reverse-transcription (5'-3') \\
\hline hsa-miR-376a & gtcgtatccagtgcagggtccgaggtattcgcactggatacgacacgtgg ACGTGG \\
hsa-miR-491-3p & gtcgtatccagtgcagggtccgaggtattcgcactggatacgacacgtggGTAGAAG \\
hsa-miR-220b & gtcgtatccagtgcagggtccgaggtattcgcactggatacgacacgtgg AAGTGT \\
hsa-miR-524-5p & gtcgtatccagtgcagggtccgaggtattcgcactggatacgacacgtggGAGAAAG \\
let7b & gtcgtatccagtgcagggtccgaggtattcgcactggatacgacacgtggAACCACA \\
hsa-miR-125a-3p & gtcgtatccagtgcagggtccgaggtattcgcactggatacgacacgtGGCTCCCA \\
hsa-miR-943 & gtcgtatccagtgcagggtccgaggtattcgcactggatacgacacgtggCTGGAGG \\
hsa-miR-649 & gtcgtatccagtgcagggtccgaggtattcgcactggatacgacacgtggGACTCTTG \\
hsa-miR-890 & gtcgtatccagtgcagggtccgaggtattcgcactggatacgacacgtggCAACTG \\
hsa-miR-628-3p & gtcgtatccagtgcagggtccgaggtattcgcactggatacgacacgtggTCGACTG \\
hsa-miR-425 & gtcgtatccagtgcagggtccgaggtattcgcactggatacgacacgtggTCAACG \\
hsa-miR-129-5p & gtcgtatccagtgcagggtccgaggtattcgcactggatacgacacgtggGCAAGC \\
hsa-miR-34a & gtcgtatccagtgcagggtccgaggtattcgcactggatacgacacgtgGACAACC \\
hsa-miR-181b & gtcgtatccagtgcagggtccgaggtattcgcactggatacgacacgtggACCCACCG \\
\hline
\end{tabular}


Table 2. Primers for real-time PCR

\begin{tabular}{lll}
\hline \multirow{2}{*}{ Gene name } & Primers for real-time PCR & \\
\cline { 2 - 3 } & Forward primer (5'-3') & Reverse primer (5'-3') \\
\hline hsa-miR-376a & AUCAUAGAGGAAAAU & GTGCAGGGTCCGAGGT \\
hsa-miR-491-3p & CUUAUGCAAGAUUCC & GTGCAGGGTCCGAGGT \\
hsa-miR-220b & CCACCACCGUGUCUG & GTGCAGGGTCCGAGGT \\
hsa-miR-524-5p & CUACAAAGGGAAGCA & GTGCAGGGTCCGAGGT \\
let7b & UGAGGUAGUAGGUUG & GTGCAGGGTCCGAGGT \\
hsa-miR-125a-3p & ACAGGUGAGGUUCU & GTGCAGGGTCCGAGGT \\
hsa-miR-943 & CUGACUGUUGCCGU & GTGCAGGGTCCGAGGT \\
hsa-miR-649 & AAACCUGUGUUGUUC & GTGCAGGGTCCGAGGT \\
hsa-miR-890 & UACUUGGAAAGGCAU & GTGCAGGGTCCGAGGT \\
hsa-miR-628-3p & UCUAGUAAGAGUGG & GTGCAGGGTCCGAGGT \\
hsa-miR-425 & AAUGACACGAUCACUCC & GTGCAGGGTCCGAGGT \\
hsa-miR-129-5p & CUUUUUGCGGUCUGGG & GTGCAGGGTCCGAGGT \\
hsa-miR-34a & UGGCAGUGUCUUAGCU & GTGCAGGGTCCGAGGT \\
hsa-miR-181b & AACAUUCAUUGCUGUC & GTGCAGGGTCCGAGGT \\
Caspase-3 & CATGGCCCTGAAATACGAAGTC & CATGGCCCTGAAATACGAAGTC \\
U6 & CTCGCTTCGGCAGCACA & AACGCTTCACGAATTTGCGT \\
GAPDH & AACGACCCCTTCATTGACCTC & CCTTGACTGTGCCGTTGAACT \\
\hline
\end{tabular}

CAGAGCCATGGGCACGTC TTCAG, right Not1 primer: gcggccgcCCTATTGCTGGATGCTTTCCAAGTCCC). The PCR product was digested with Xho1 and Not1, followed by insertion into the multiple cloning region located at the 3 '-UTR of the synthetic Renilla luciferase gene within the psiCheck2 plasmid. The psiCheck 2 plasmid also contains a synthetic firefly luciferase gene that serves as the transfection control. The retinoblastoma cells were co-transfected with the psiCheck2 vector containing caspase-3 3'-UTR and miR-376a mimic using Lipofectamine 2000 (Invitrogen, Carlsbad, CA, USA), and the co-transfection with non-targeting negative control RNA was performed as control. The cells were harvested 24 $\mathrm{h}$ after transfection, and luciferase activity was measured with a dual luciferase reporter assay kit (Promega, Madison, WI, USA) on a luminometer (Lumat LB9507).

Western blot analysis. The retinoblastoma cells were lysed with RIPA buffer (Beyotime, Jiangsu, China), sonicated on ice, and protein concentration was determined using the bicinchoninic acid kit (Beyotime, Jiangsu, China). $20 \mu \mathrm{g}$ of total protein were electrophoresed on a $10 \%(w / v)$ SDS-PAGE gel, transferred onto nitrocellulose membrane and blocked with $5 \%(\mathrm{w} / \mathrm{v})$ non-fat dried milk. Membranes were probed with the caspase- 3 primary antibody (1:200), followed by the peroxidase-conjugated secondary antibody. Protein signal was visualized by chemiluminescence reagents under a GeneGnome Bio Imaging System (Syngene, MD,USA). The amount of full-length and cleaved caspase- 3 was quantified by densitometry and normalized to GAPDH, an internal standard.

Statistical analysis. Statistical analyses were carried out using Student's $t$ test between two groups or ANOVA followed by post hoc comparisons among three or more groups. All data provided here are mean \pm SD. $P<0.05$ was considered significant.

\section{Results}

1. Effect of ATO on induction of apoptosis in retinoblastoma cells. Retinoblastoma cells were treated with ATO for 72 hours, and apoptosis was detected by AV/PI dual staining. As shown in Figure.1, ATO $(0.5,1,2,4,8 \mu \mathrm{M})$ dose-dependently promoted the apoptosis of retinoblastoma cells. Apoptotic

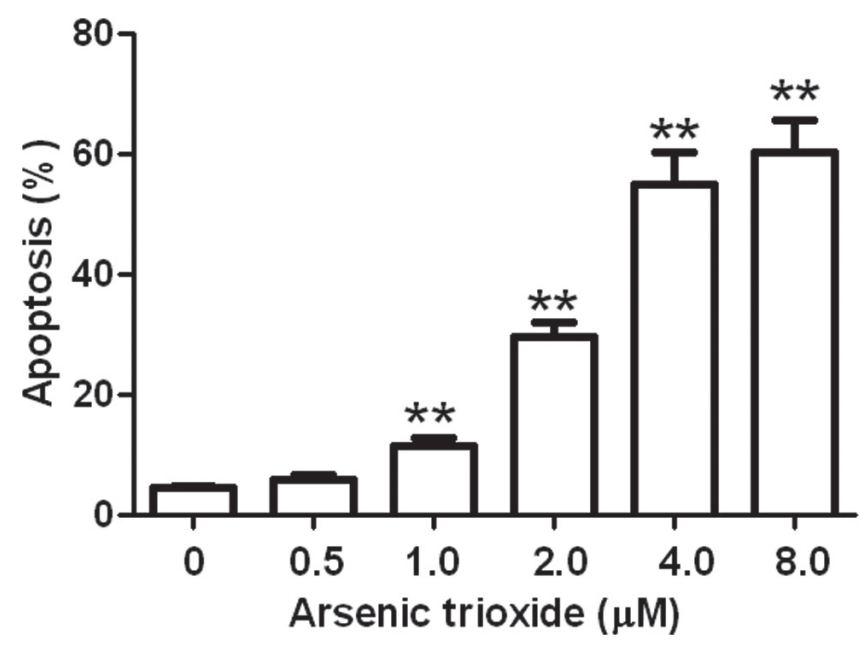

Figure.1 ATO dose-dependently promoted the apoptosis of retinoblastoma cells. Retinoblastoma cells were treated with ATO for 72 hours, and apoptosis was detected by AV/PI dual staining. ATO $(0.5-8 \mu \mathrm{M})$ dose-dependently promoted the apoptosis of retinoblastoma cells. ${ }^{\star *} \mathrm{P}<0.01 \mathrm{vs}$ control. 
Table 3. Aberrant expression of miRNAs in retinoblastoma cells subjected to ATO

\begin{tabular}{lcc}
\hline \multirow{2}{*}{ MicroRNA } & \multicolumn{2}{c}{ Fold change } \\
\cline { 2 - 3 } & Microarray & Real-time PCR \\
\hline hsa-miR-376a & $-6.6^{* *}$ & $-7.2^{* *}$ \\
hsa-miR-491-3p & $-3.2^{* *}$ & $-2.9^{* *}$ \\
hsa-miR-220b & $-3.0^{* *}$ & $-2.0^{* *}$ \\
hsa-miR-524-5p & $-2.7^{* *}$ & $-2.0^{* *}$ \\
let7b & $-2.2^{*}$ & -1.4 \\
hsa-miR-125a-3p & $3.8^{* *}$ & $3.2^{* *}$ \\
hsa-miR-943 & $3.8^{* *}$ & $2.8^{* *}$ \\
hsa-miR-649 & $3.5^{* *}$ & $3.1^{* *}$ \\
hsa-miR-890 & $3.4^{* *}$ & $2.6^{* *}$ \\
hsa-miR-628-3p & $2.9^{* *}$ & $2.4^{* *}$ \\
hsa-miR-425 & $2.7^{* *}$ & $2.2^{* *}$ \\
hsa-miR-129-5p & $2.4^{*}$ & $2.0^{* *}$ \\
hsa-miR-34a & $2.1^{*}$ & 1.3 \\
hsa-miR-181b & $2.1^{*}$ & $2.4^{* *}$ \\
\hline
\end{tabular}

${ }^{*} \mathrm{P}<0.05,{ }^{* *} \mathrm{P}<0.01$ vs.control

rate in ATO treated groups at the five concentrations were $6.0 \pm 0.7 \%, 11.6 \pm 1.3 \%, 29.6 \pm 2.4 \%, 54.9 \pm 5.3 \%, 60.3 \pm$ $5.4 \%$, respectively. The apoptotic rate of higher concentration groups $(1-8 \mu \mathrm{M})$ were all significantly higher than that of control group $(4.4 \pm 0.5 \%, P<0.01) .4 \mu \mathrm{M}$ ATO induced obvious apoptosis and the dose is not too high, so we used 4 $\mu \mathrm{M}$ ATO in the further study.

2. MiRNA expression profiles in retinoblastoma cells treated with ATO. MiRNA microarray analysis showed that 14 miRNAs were regulated by at least 2 -fold in retinoblastoma cells treated with $4 \mu \mathrm{M}$ ATO, compared to the control group

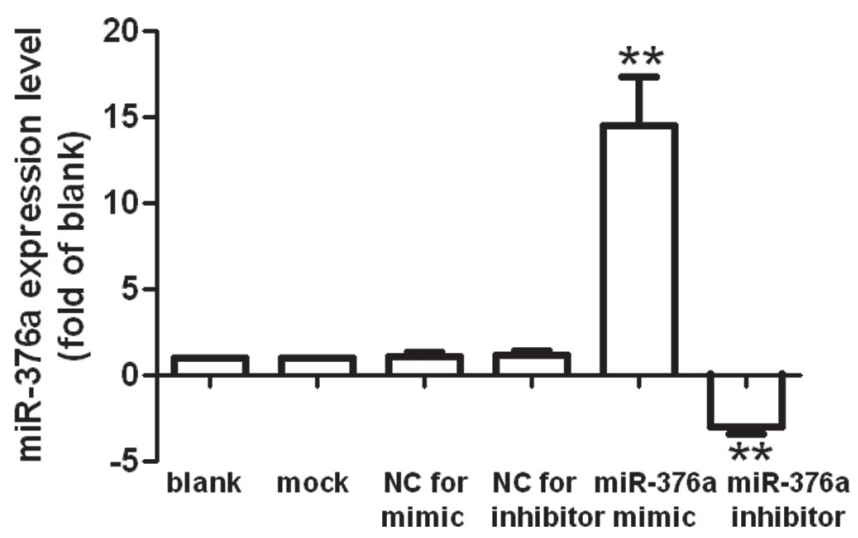

Figure.2 MiR-376a levels in retinoblastoma cells transfected with miR-376a mimic or inhibitor. Cells were transfected with $50 \mathrm{nM}$ of miR-376a mimic or inhibitor for $\mathbf{2 4} \mathrm{h}$, with the aid of transfection agent. Mock transfection (transfection without RNA) and non-targeting negative control RNA were used as controls. MiR-376a levels were determined using real-time RT-PCR, normalized to U6, and expressed as the fold change of the control. Data are presented as the means \pm SD from three independent experiments $\left({ }^{*} \mathrm{P}<0.01\right.$ vs. blank)
(Table 3). Among them, miR-376a, -491-3p, -220b, -524-5p and - let7b were down-regulated, whereas miR-125a-3p, -943, $-649,-890,-628-3 p,-425,-129-5 p,-34 a$, and $-181 b$ were upregulated. The abovementioned miRNAs aberrantly expressed in retinoblastoma cells treated with $4 \mu \mathrm{M}$ ATO were mostly verified by real-time RT-PCR (Table 1). MiR-376a was selected for further functional studies because of its remarkable change in response to ATO and bioinformatic prediction of potential target genes associated with apoptosis.

3. MiR-376a expression was regulated by its mimic and inhibitor. In cultured retinoblastoma cells, transfection of miR-376a mimic or inhibitor with the aid of transfection agents significantly increased or decreased the level of miR376a (Figure. 2). The dose of $50 \mathrm{nM}$ was identified to be optimal for both the mimic and inhibitor of miR-376a during preliminary experiments. As shown in Figure. 2, neither mock nor negative control RNA transfection affected miR-376a expression in cultures. $50 \mathrm{nM}$ miR-376a mimic and inhibitor up- or down-regulated miR-376a by $14.5 \pm 2.8$ and $3.0 \pm 0.4$ fold of blank (both $\mathrm{P}<0.01$ ), respectively.

4. Effect of miR-376a on apoptosis in retinoblastoma cells. As shown in Figure.3, the rate of apoptosis in normal cultured retinoblastoma cells was approximately $5 \%$. Mock, control RNA or miR-376a mimic transfection did not alter baseline apoptosis. By contrast, the miR-376a inhibitor resulted in a much higher rate of apoptosis $(31.2 \pm 5.4 \%, \mathrm{P}<0.01)$. We further investigated the effects of miR-376a on ATO-induced apoptosis in retinoblastoma cells. As shown in Figure.4, miR-376a mimic decreased ATO-induced apoptosis. By contrast, miR-376a inhibitor further aggravated ATO-induced apoptosis.

5. Caspase-3 is a target of miR-376a. To explore the molecular mechanism, we searched for potential targets of miR-376a using bioinformatic algorithms such as MiRanda (August 2010 Release, http://www.microrna.org), Pictar 


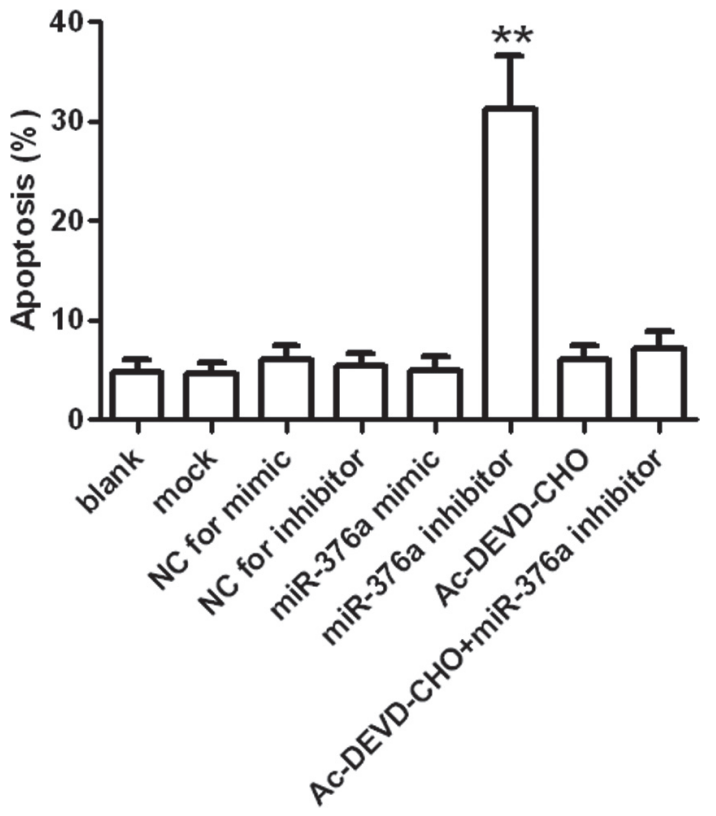

Figure.3 Apoptosis detected by AV/PI dual staining in retinoblastoma cells transfected with miR-376a mimic or inhibitor. The procedure for RNA transfection was the same as that described in Fig. 2. The caspase-3 inhibitor Ac-DEVD-CHO was added into the medium at a final concentration of $10 \mu \mathrm{M} .{ }^{* *} \mathrm{P}<0.01 \mathrm{vs}$. blank.

(March 2007 Release, http://pictar.mdc-berlin.de/) and TargetScan (Release 6.2, http://www.TargetScan.org). Caspase-3, a critical executioner of apoptosis, was predicted as a putative target of miR-376a. One miR-376a-binding site was identified within the 3'-UTR of caspase-3 mRNA (Fig. 5A).

To examine whether miR-376a regulates caspase- 3 expression, we generated a luciferase reporter encoding caspase- 3 3'-UTR that contains miR-376a binding sites. This reporter was co-transfected with miR-376a mimic into retinoblastoma cells, and the luciferase activity assay was performed $24 \mathrm{~h}$ after transfection. Compared with the negative control RNA, the miR-376a mimic significantly suppressed the activity of the luciferase reporter fused with caspase-3 3'-UTR by 35\% (Figure. $5 \mathrm{~B}$ ), suggesting the inhibition of miR-376a on caspase-3 expression through its 3'-UTR. Additionally, we determined both the mRNA and protein levels of caspase-3. As shown in Figure. 5C-E, the mRNA and protein levels of caspase-3 were exclusively increased by $4 \mu \mathrm{M}$ ATO. No changes of caspase- 3 mRNA were detected in normal solution or ATO-treated cells transfected with the miR-376a mimic or inhibitor. However, remarkable increases of caspase- 3 proteins were observed in cells receiving the miR-376a inhibitor (Figure. 5D and E). Furthermore, miR-376a mimic significantly decreased caspase-3 proteins. These results indicate that miR-376a inhibits the synthesis of caspase- 3 proteins, likely through post-transcriptional mechanisms. Furthermore, as shown in Figure 3 and Figure 4, the increased apoptosis of retinoblastoma cells

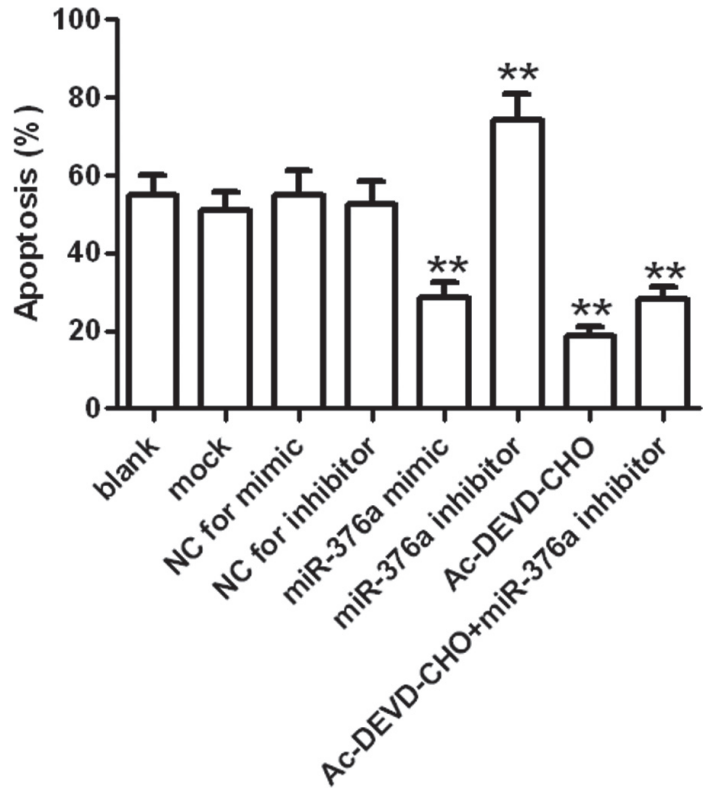

Figure.4 ATO induced apoptosis detected by AV/PI dual staining in retinoblastoma cells transfected with miR-376a mimic or inhibitor. The procedure for RNA transfection was the same as that described in Fig. 2. The caspase- 3 inhibitor Ac-DEVD-CHO was added into the medium at a final concentration of $10 \mu \mathrm{M} 20 \mathrm{~min}$ prior to administration of $4 \mu \mathrm{M}$ ATO. ${ }^{\star *} \mathbf{P}<0.01$ vs. blank.

caused by the miR-376a inhibitor was abolished by $10 \mu \mathrm{M}$ Ac-DEVD-CHO, a caspase-3 inhibitor, suggesting that miR376a involved in ATO-induced apoptosis in retinoblastoma cells by caspase- 3 .

\section{Discussion}

In the present study, we demonstrated that ATO induced apoptosis in retinoblastoma cells by decreasing expression of miR-376a, in association with increased expression of caspase3 , which is a target of miR-376a.

The accumulating evidences indicated that ATO could regulate many biological functions such as cell proliferation and apoptosis in retinoblastoma cells [7]. Apoptosis is an important mode of cell death that occurs in response to a variety of agents including ionizing radiation or anticancer chemotherapeutic drugs [13]. Several lines of evidence suggest that interleukin-1-converting enzyme (ICE)/caspase family plays a crucial role in apoptosis $[14,15]$. Especially, caspase3 has been shown to be a key component of the apoptotic machinery.

MiRNAs are approximately 22-nucleotide endogenous RNAs that exert profound biological effects. MiRNAs are usually post-transcriptional regulators that bind to partly complementary sequences in the 3'-UTR of target mRNAs, resulting in translational repression $[16,17]$. It is estimated that over 1000 miRNAs are encoded in the human genome, 
A

miR-376a 3'-UGCACCUAAAAGGAGAUACUA-5' Caspase hsa 5'-UACAGUGAUGCUGUGCUAUGAAU-3'

B

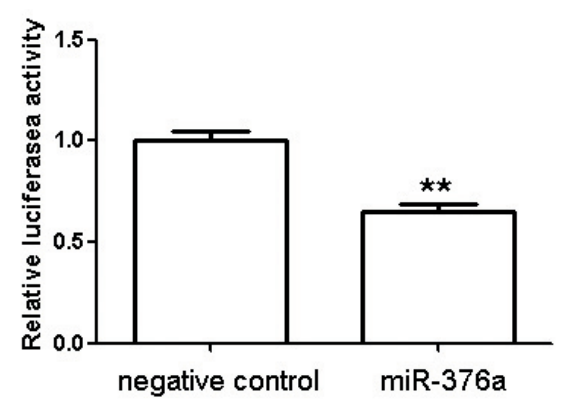

C

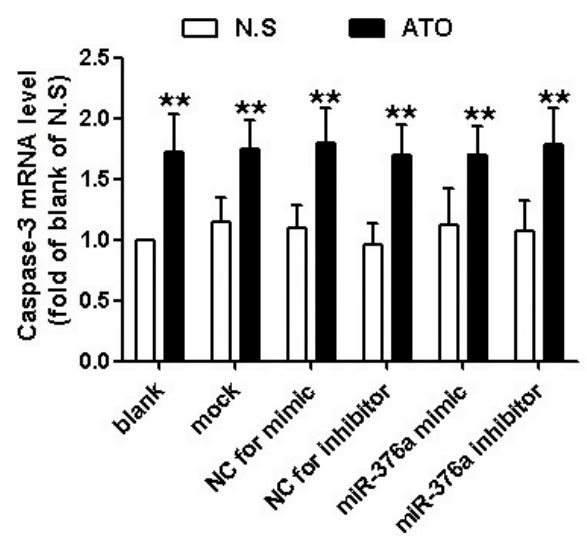

D

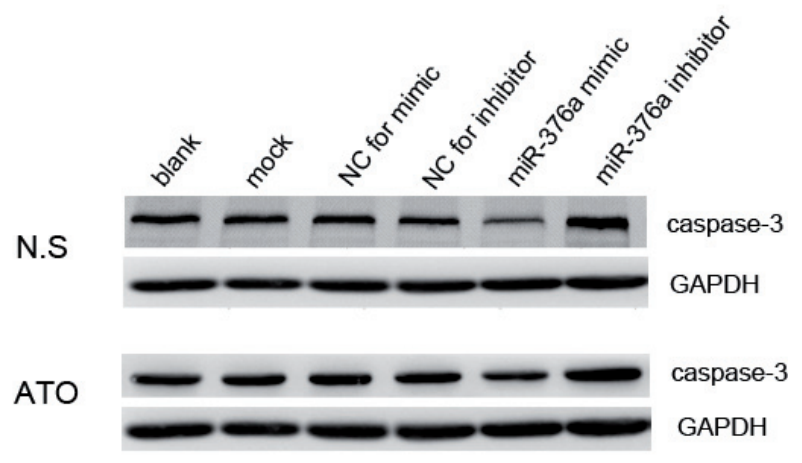

E

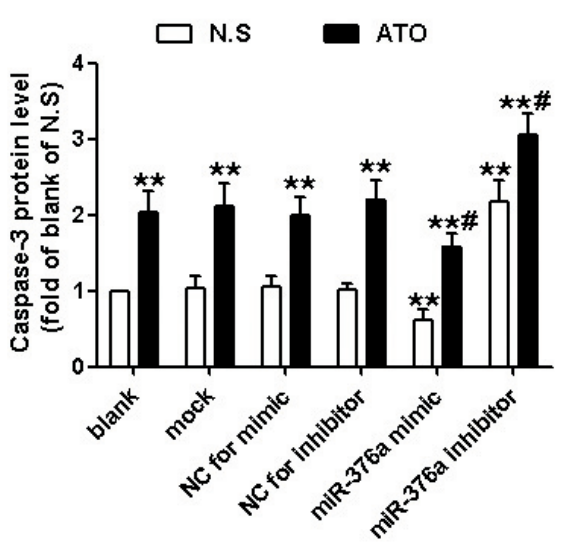

Figure.5 Caspase-3 is a target of miR-376a. A, The potential binding site between miR-376a and the 3' UTR of caspase-3 mRNA. The complementary nucleotides between miR-376a and the target region of caspase-3 3'-UTR are connected with short vertical lines. B, Luciferase reporter assay was performed by co-transfection of luciferase reporter containing 3'-UTR of caspase-3 with miR-376a mimic into retinoblastoma cells. Luciferase activity was determined $24 \mathrm{~h}$ after transfection. C, Caspase- $3 \mathrm{mRNA}$ levels determined by real-time PCR. The PCR data were normalized to GAPDH, and expressed as the fold change of the blank of normal solution (N.S). D, Representative western blot image for caspase-3. E, The quantitative data of caspase-3 protein levels determined by western blot. ${ }^{* *} \mathrm{P}<0.01$ vs. blank of N.S; \# $\mathrm{P}<0.05$ vs. blank of ATO.

and they target approximately $60 \%$ of mammalian genes [18]. Accumulating evidence has shown that miRNAs can function as oncogenes or tumor suppressors involved in cancer development, including tumor metastasis and proliferation $[17,19]$.

In the present study, the miRNA microarray analysis identified 14 miRNAs in the retinoblastoma cells treated by ATO changing more than 2-fold compared to the control group. Among them, the up-regulation of miR-125a-3p, -943, -649, $-890,-628-3 p,-425,-129-5 p$ and $-181 b$, and the down-regulation of miR-376a, -491-3p, -220b and -524-5p were confirmed by real-time PCR. A major finding of the present study is that low expression of miR-376a significantly promoted apoptosis of retinoblastoma cells by increasing caspase- 3 protein levels. Based on bioinformatic algorithms, many genes were included as potential targets of miR-376a. Strikingly, caspase-3 is one of them. The luciferase reporter assay in this study confirmed caspase- 3 to be a target of miR-376a. Caspase- 3 is a well-established executioner of apoptosis. In the present study, we observed that miR-376a overexpression significantly reduced protein levels of caspase-3, but did not affect mRNA levels of caspase-3, suggesting that miR-376a inhibits caspase-3 expression at the level of postatranscription, which is one of the major patterns of miRNA 
action [20]. Recently, Su et al reported there was functional complementation between transcriptional methylation regulation and post-transcriptional microRNA regulation in the human genome[21], so DNA methylation may be another mechanism besides post-transcriptional.

In summary, our study is the first to show the ATO induced apoptosis in retinoblastoma cells by unnormal expression of miRNAs. Moreover, we identified the regulatory effects of miR-376a on caspase-3 expression, indicating a potential role of miR-376a in other disorders associated with apoptosis.

Acknowledgements: This work was supported by grants from the National Natural Science Foundation of China (Nos. 30801268 and 81270003).

\section{References}

[1] N.A. LAURIE, C.S. SHIH, M.A. DYER, Targeting MDM2 and MDMX in retinoblastoma. Curr Cancer Drug Targets 7 (2007) 689-695. http://dx.doi.org/10.2174/156800907782418266

[2] E. LENGFELDER, W.K. HOFMANN, D. NOWAK, Impact of arsenic trioxide in the treatment of acute promyelocytic leukemia. Leukemia 26 (2012) 433-442. http://dx.doi. org/10.1038/leu.2011.245

[3] F. BINET, F. ANTOINE, D. GIRARD, Interaction between arsenic trioxide and human primary cells: emphasis on human cells of myeloid origin. Inflamm Allergy Drug Targets 8 (2009) 21-27. http://dx.doi.org/10.2174/187152809787582516

[4] I. ORA, L. BONDESSON, C. JONSSON, J. LJUNGBERG, I. PORN-ARES, et al. Arsenic trioxide inhibits neuroblastoma growth in vivo and promotes apoptotic cell death in vitro. Biochem Biophys Res Commun 277 (2000) 179-185.

[5] L.E. BECKER, D. HINTON, Primitive neuroectodermal tumors of the central nervous system. Hum Pathol 14 (1983) 538-550. http://dx.doi.org/10.1016/S0046-8177(83)80006-9

[6] M.A. DYER, Mouse models of childhood cancer of the nervous system. J Clin Pathol 57 (2004) 561-576. http://dx.doi. org/10.1136/jcp.2003.009910

[7] J.H. KIM, Y.S. YU, D.H. KIM, C.J. KIM, K.W. KIM, Antitumor activity of arsenic trioxide on retinoblastoma: cell differentiation and apoptosis depending on arsenic trioxide concentration. Invest Ophthalmol Vis Sci 50 (2009) 1819-1823.

[8] A. ESQUELA-KERSCHER, F.J. SLACK, Oncomirs - microRNAs with a role in cancer. Nat Rev Cancer 6 (2006) 259-269. http://dx.doi.org/10.1038/nrc1840

[9] O.A. KENT, J.T. MENDELL, A small piece in the cancer puzzle: microRNAs as tumor suppressors and oncogenes. Oncogene 25 (2006) 6188-6196. http://dx.doi.org/10.1038/ sj.onc. 1209913
[10] J.C. HUANG, T. BABAK, T.W. CORSON, G. CHUA, S. KHAN et al. Using expression profiling data to identify human microRNA targets. Nat Methods 4 (2007) 10451049.

[11] S.J. MARTIN, C.P. REUTELINGSPERGER, A.J. MCGAHON, J.A. RADER, R.C. VAN SCHIE, et al. Early redistribution of plasma membrane phosphatidylserine is a general feature of apoptosis regardless of the initiating stimulus: inhibition by overexpression of Bcl-2 and Abl. J Exp Med 182 (1995) 1545-1556. http://dx.doi.org/10.1084/ jem.182.5.1545

[12] I. VERMES, C. HAANEN, H. STEFFENS-NAKKEN, C. REUTELINGSPERGER, A novel assay for apoptosis. Flow cytometric detection of phosphatidylserine expression on early apoptotic cells using fluorescein labelled Annexin V. J Immunol Methods 184 (1995) 39-51. http://dx.doi. org/10.1016/0022-1759(95)00072-I

[13] D.E. FISHER, Apoptosis in cancer therapy: crossing the threshold. Cell 78 (1994) 539-542. http://dx.doi.org/10.1016/ 0092-8674(94)90518-5

[14] G.M. COHEN, CASPASES: the executioners of apoptosis. Biochem J 326 (Pt 1) (1997) 1-16.

[15] B.G. CAMPLING, J. PYM, P.R. GALBRAITH, S.P. COLE, Use of the MTT assay for rapid determination of chemosensitivity of human leukemic blast cells. Leuk Res 12 (1988) 823-831. http://dx.doi.org/10.1016/0145-2126(88)90036-7

[16] D.P. BARTEL, MicroRNAs: target recognition and regulatory functions. Cell 136 (2009) 215-233. http://dx.doi.org/10.1016/ j.cell.2009.01.002

[17] D.P. BARTEL, MicroRNAs: genomics, biogenesis, mechanism, and function. Cell 116 (2004) 281-297. http://dx.doi. org/10.1016/S0092-8674(04)00045-5

[18] R.C. FRIEDMAN, K.K. FARH, C.B. BURGE, D.P. BARTEL, Most mammalian mRNAs are conserved targets of microRNAs. Genome Res 19 (2009) 92-105. http://dx.doi. org/10.1101/gr.082701.108

[19] Q. DONG, P. MENG, T. WANG, W. QIN, F. WANG, et al. MicroRNA let-7a inhibits proliferation of human prostate cancer cells in vitro and in vivo by targeting E2F2 and CCND2. PLoS One 5 (2010) e10147. http://dx.doi.org/10.1371/journal. pone.0010147

[20] R.S. PILLAI, S.N. BHATTACHARYYA, W. FILIPOWICZ, Repression of protein synthesis by miRNAs: how many mechanisms? Trends Cell Biol 17 (2007) 118-126. http://dx.doi. org/10.1016/j.tcb.2006.12.007

[21] Z. SU, J. XIA, Z.ZHAO, Functional complementation between transcriptional methylation regulation and post-transcriptional microRNA regulation in the human genome. BMC Genomics 12 Suppl 5 (2011) S15. http://dx.doi.org/10.1186/ 1471-2164-12-S5-S15 